\title{
When Records Are Expectations How to Project Rankings for Annual Global Temperatures
}

\author{
Adapted from "Should We Expect \\ Each Year in the Next Decade \\ (2019-28) to Be Ranked among the \\ Top 10 Warmest Years Globally?" \\ by Anthony Arguez (NOAA/ \\ NCEI), Shannan Hurley, Anand \\ Inamdar, Laurel Mahoney, Ahira \\ Sanchez-Lugo, and Lilian Yang. \\ Published online in BAMS, May \\ 2020. For the full, citable article, \\ see DOI:10.1175/BAMS-D-19-0215.1.
}

T he annual rankings of global temperature are widely cited by media as a first-order metric of recent climate change, easily understood by the general public. According to observations from NOAA's Merged Land Ocean Global Surface Temperature Analysis Dataset (NOAAGlobalTemp) 5.0, the most recent 5 years (2014-18) comprise the 5 warmest years on record, with 2018 being the fourth-warmest globally since 1880 (earliest reading available). The warmest year was 2016. Given the record or near-record heat of recent years, should we expect each year in the next decade (2019-28) to be ranked among the top-10 warmest years globally?

To answer this question, we analyze the monthly version of NOAAGlobalTemp to project future end-of-year annual global rankings. In addition, given that nearly every recent year (at least initially) is among the top-10 warmest, we introduce a "temperature score" to help NOAA communicate the coolness or warmth of a given year relative to the long-term trend. We believe this is the first such projected ranking and temperature score currently produced operationally. Our objective is to use this tool to improve the communication of climate change impacts to the general public. 


\section{Methodology}

The purpose here is to select an algorithm for projecting rankings of global annual temperatures. We use the monthly global temperatures from the NOAAGlobalTemp time series for January 1975 through December 2018. ${ }^{1}$ These global temperatures are averages of surface air temperatures from approximately 26,000 landbased stations, plus ship-, buoy-, and float-based sea surface (water) temperatures, all quality controlled and corrected, taking into account sea ice concentrations, among other factors. The data are averaged for each month at each point on $a 5^{\circ} \times 5^{\circ}$ global grid, and each grid point is weighted to account for the variation of spacing by latitude before being combined into a global average.

This dataset gives us a 40+-yr baseline with which to estimate the month-to-month past fluctuations-likely to be representative in real timeeven as global temperatures show a stable upward trend since the mid-1970s. The past fluctuations are estimated with

\begin{tabular}{|c|c|c|c|c|}
\hline Ranking & Year & Anomaly & Running ranking & Temperature score \\
\hline 1 & 2016 & 0.675 & 1 & 10 \\
\hline 2 & 2015 & 0.615 & 1 & 10 \\
\hline 3 & 2017 & 0.590 & 3 & 9 \\
\hline 4 & 2018 & 0.507 & 4 & 5 \\
\hline 5 & 2014 & 0.423 & 1 & 4 \\
\hline 6 & 2010 & 0.409 & 1 & 7 \\
\hline 7 & 2013 & 0.359 & 2 & 2 \\
\hline 8 & 2005 & 0.355 & 1 & 8 \\
\hline 9 & 1998 & 0.340 & 1 & 10 \\
\hline 10 & 2009 & 0.330 & 3 & 4 \\
\hline
\end{tabular}
autoregressive (AR) modeling using the residuals left in the data after extracting the long-term trends.

We experimented with three different methods, each performed separately for the land and ocean data (which are later combined). In the first case, "AR modeling without trend extension," we incorporate the trend for observed years but do not apply it to the projection of the future decade-(i.e., imposing a mean future trend of zero). In the second case, "AR with trend extension," effectively assumes the mean observed trend continues during the 10-yr forecast period. Comparing these two cases allows us to characterize the skill associated with the trend itself. The third case, "AR+ENSO," offsets the trend extension approach by an adjustment based on real-time ENSO conditions. (The Arctic Oscillation and Atlantic multidecadal oscillation can impact global surface temperatures, but the effects are small over the time interval of our study.) We utilize the oceanic Niño index (ONI), a 3-month running average of SST anomalies in the Niño 3.4 region. $^{2}$ This adjustment is determined by analysis that shows maximal correlation between the most recent ONI value and the running mean of NOAAGlobalTemp residuals at lags of 1-4 months.

We use 1999-2018 as a 20-yr reforecasting period to test three methodologies for projecting the rankings of annual global temperatures and anomalies. This reforecasting test compares projections to observed rankings. Each successive month in the reforecasting

\footnotetext{
/noaa-global-surface

-temperature

-noaaglobaltemp

1 https://www.ncdc

.noaa.gov/data-access Imarineocean-data
} 
most recent month of observed data available to make an operational projection in January 1999. This approach is repeated for each month from January 1999 through December 2018.

In this study, we are focused on the "running ranking." This is the ranking of an individual year versus all prior years (i.e., when the year is newly ended, not the ranking it now has, in the additional perspective of the ensuing years). Since virtually all newly ended years since 1988 would have had a top-10 running ranking, it would be useful to distinguish between warmer and colder years relative to the sustained long-term trend to communicate climate monitoring impacts. For example, the years 2008 and 2011 were considerably cooler than surrounding years and below the overall trend line, whereas 1998 and 2016 were not only considered the warmest years on record but were also warmer than surrounding years. To provide such context relative to long-term trends, we propose a simple new annual "temperature score" from 1 (a very cold year) to 10 (very warm).

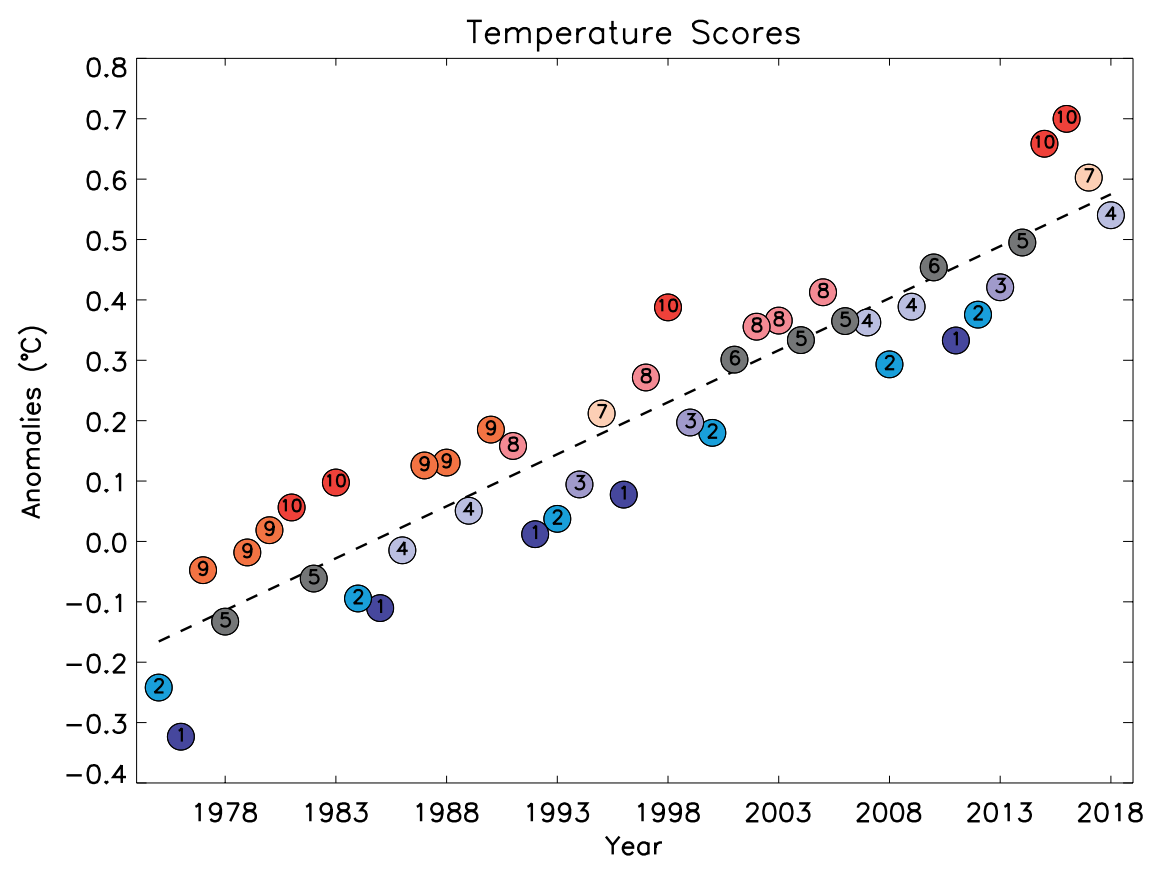

$\Delta$ * Global annual temperature scores based on the annual NOAAGlobalTemp time series from 1975-2018. The ordinate indicates the NOAAGlobalTemp anomaly relative to the 1971-2000 base period. The inscribed value indicates the temperature score and the dashed line indicates the OLS regression line for 1975-2018.

Projected (median) rankings for 2019-28 based on the NOAAGlobalTemp dataset from 1975 to 2018 for the AR without trend extension and AR with trend extension methods. The $95 \%$ prediction intervals are indicated by the $2.5 \%$ and $97.5 \%$ levels of the Monte Carlo simulations. $\frac{*}{\nabla}$

\begin{tabular}{|c|c|c|c|c|c|c|}
\hline \multirow{2}{*}{ Year } & \multicolumn{3}{|c|}{ AR without trend extension } & \multicolumn{3}{c|}{ AR with trend extension } \\
\cline { 2 - 7 } & $2.5 \%$ level & Median & $\mathbf{9 7 . 5 \% \text { level }}$ & $\mathbf{2 . 5 \%}$ level & Median & $\mathbf{9 7 . 5 \%}$ level \\
\hline 2019 & 1 & 4 & 7 & 1 & 4 & 7 \\
\hline 2020 & 1 & 5 & 8 & 1 & 4 & 8 \\
\hline 2021 & 1 & 5 & 10 & 1 & 4 & 9 \\
\hline 2022 & 1 & 6 & 12 & 1 & 4 & 9 \\
\hline 2023 & 1 & 6 & 13 & 1 & 4 & 9 \\
\hline 2024 & 1 & 7 & 14 & 1 & 4 & 10 \\
\hline 2025 & 1 & 7 & 15 & 1 & 4 & 11 \\
\hline 2026 & 1 & 8 & 16 & 1 & 3 & 12 \\
\hline 2027 & 1 & 8 & 17 & 1 & 3 & 12 \\
\hline 2028 & 1 & 9 & 18 & 1 & 3 & 13 \\
\hline
\end{tabular}




\section{Results}

During the monitoring year (the first of the 10-yr forecast period), all three methods perform similarly. During outlook years (the next 9 years), there are no appreciable differences between the AR+ENSO and the AR with trend extension. Both of these methods far outperform the AR without trend extension approach for years 5 through 10 . Notably, the very strong El Niño events of 1982/83, 1997/98, and 2015/16 are associated with temperature scores of 10 (in 1983, 1998, and 2016, respectively), indicating exceptionally warm years relative to the trend. In contrast, the strong La Niña events of 2007/08 and 2010/11 both have temperature scores of 1 (in 2008 and 2011, respectively) though they were over $0.2^{\circ} \mathrm{C}$ warmer than 1983 (running ranking of 1 and temperature score of 10) and are warmer than all years prior to 1998. More recently, the years 2014 and 2013 were initially ranked as the first and second warmest years on record, respectively, yet their corresponding temperature scores are 4 and 2, respectively, on the colder side of the trend line. Thus, what we consider to be a "warm year" or "cold year" has changed over time.

\section{Discussion and conclusions}

Based on our results, we propose using the AR with trend extension approach operationally for characterizing the annual ranking probabilities during the course of the monitoring year as well as for the outlook years. Projections of the next 10 years using NOAAGlobalTemp data through December 2018 suggest a greater than $99 \%$ probability that most of the years between 2019 and 2028 will also be top-10 warmest years $(75.3 \%$ probability that every year will meet this criterion). It would likely take an abrupt climate shift for even a few years within the next decade to register outside the top-10 warmest years.

Given the strong likelihood for future years to remain near record levels, we recommend that global monitoring analyses incorporate the temperature score to better communicate the differentiation of warmer and colder years relative to the long-term trend. Taken in tandem, the new approaches for temperature scores and projected rankings help the general public characterize global temperature in context.

\section{METADATA}

BAMS: What would you like readers to learn from this article?

Anthony Arguez (NOAA/NCEI): I would like the general public to know that there is not a great deal of suspense: most years-if not all-over the next decade will likely register as top 10 warmest years. In fact, the data suggest we should expect this, as it would likely take a pretty abrupt change to get us off this trajectory.

BAMS: This is a novel way at looking at climate expectations. What drives your interest in communicating about climate data?

AA: I feel like I've been staring at the annual global temperature time series continually over the past 15 years or so because it is just so interesting in many ways. I find it challenging and rewarding to develop methods to translate volumes of data into answers to specific questions posed by the general public. I've drawn inspiration from Nate Silver, whose penchant for expounding on and communicating the "signal" hidden in the "noise" informs the way I would like to see myself and fellow climate scientists communicate to the general public more effectively.

BAMS: What surprises/surprised you the projected rankings?

AA: I was very surprised that the ranking errors we found were so small! Before calculating the results, I had a gut feeling that these errors would be modest, but the mean absolute ranking error of $\sim 2$ spots a full 10 years out was well below anything I could have imagined. I clearly underappreciated the predictability inherent in the observed upward trend when it comes to annual global temperature rankings.

BAMS: What was the biggest challenge you encountered in this paper?

AA: I think the biggest challenge we faced was that we were not aware of any similar operational products in existence (neither for projected rankings or global annual temperature scores), or of any papers that had characterized ranking errors in a similar fashion, so we were in uncharted territory to some extent.

BAMS: What's next?

AA: I am still staring at the annual global temperature time series, and hope to publish new papers. 




